ISSN 1817-3721, E-ISSN 1818-8745

- Short communication

Plant Tissue Cult. \& Biotech. 29(1): 121-126, 2019 (June)

(CBangladesh Assoc. for Plant Tissue Culture \& Biotechnology



\title{
Use of Chitosan as Low Cost Source to Overcome Endophytic Contaminations under In vitro Conditions
}

\author{
Neeta Shivakumar* and Manasa N Hegde \\ Department of Biotechnology, RV College of Engineering, RV Vidyanikethan Post, Mysuru Road, \\ Bengaluru-560059, Karnataka, India \\ Key words: Chitosan, In vitro Culture, Endophytic contamination, Vigna radiata
}

Contamination is a serious problem that causes severe loss of in vitro grown cultures. This problem becomes even more acute when the contaminations are of endophytic origin. Antibiotics are used to avoid such contaminations. Using costly antibiotics indirectly increases the cost of tissue culture. So, it was intended to use chitosan in tissue culture whose antimicrobial properties are already known. Effect of chitosan in in vitro culture has not been studied for endophytic contaminants. Different concentrations of chitosan $(5,10,15,20 \mathrm{mg} \Lambda$, respectively) were added to the media and in vitro culture of Vigna radiata seeds were carried out. Media without chitosan was used as control to compare the growth. All the experiments were carried out in triplicates. $15 \mathrm{mg} /$ of chitosan was proven to be the best against endophytic organisms in in vitro culture of Vigna radiata. Hence, the authors propose the use of chitosan as a low cost substitute for antibiotics to avoid endophytic contaminations for in vitro cultures.

Many of the microorganisms are likely to be present inter cellularly and intracellularly. Intercellular contaminations can be avoided by surface sterilization techniques and disinfectants (Cassells, 1991). Antibiotics or combination of antibiotics like ampicillin, tetracyclin, erythromycin, gentamycin and rifampicin are mainly used to avoid endophytic contaminations in the media. Use of these costly antibiotics indirectly increases the cost of tissue culture. There are many disadvantages of using antibiotics like high concentration might damage the plants and cause genetic variations. Seeds are prone to have endophytic contaminants and hence used as explants for in vitro culture (Falkiner 1997, Kneifel and Leonhardt 1992).

Chitin is mainly found in the exoskeleton of arthropods, the beaks of cephalopods, eggs and gut linings of nematodes. Various microbes also produce chitin in cell walls, membranes and spores. Chitin shares a number of biochemical similarities with the

*Author for correspondence: <neeta@rvce.edu.in>.

DOI: https://doi.org/10.3329/ptcb.v29i1.41984 
Cellulose found in plant cell walls. Chitosan is a highly basic polysaccharide, with varying nitrogen content. Chitosan solutions are highly stable over a long period (Sharp, 2013). In order to take care for the increased cost in vitro cultivation an effort has been made to substitute the costly antibiotics with the low cost and easily available chitosan for the in vitro culture.

Chitosan shows varieties of antimicrobial activities which depends on the type of chitosan, degree of polymerization, chemical composition and environmental conditions. The purity of chitosan influences its toxicological profile (Raafat et al., 2009). Chitosan is an important regulatory component in plant fungal interaction. It can increase many plant's defense responses by eliciting synthesis of phytoalexins, cell wall phenol, and callus. It can also directly inhibit the growth of a wide range of fungi. The interaction between poly cationic chitosan and electronegative residues at the cell surface alters cell permeability, causing the leakage of intracellular electrolytes, protein, and other cell components (Ahmed et al., 1992). Since a large amount of the crustacean exoskeleton is readily available as a by-product of the seafood processing industry, the raw material for chitosan production is relatively inexpensive, and thereby the production of chitosan on a large scale from this renewable bio-resource is economically feasible. Chitosan has been 'generally recognized as safe' (GRAS) by US FDA (Raafat et al., 2009). So, it was intended to use chitosan in in vitro culture to avoid microbial contaminations. Hence, chitosan was used for in vitro culture of Vigna radiata to check its efficacy against endophytic contaminations.

The experiment was conducted in Plant Tissue Culture Laboratory at R V College of Engineering Bangalore. Vigna radiata seeds were collected from the local market of Bangalore, India and stored under cool and dry conditions. The seeds were washed and soaked overnight in water at room temperature $\left(25-34^{\circ} \mathrm{C}\right)$. Seeds were washed with 2 - 3 drops of Tween 20 under running tap water for $5 \mathrm{~min}$ and the seeds were kept in $70 \%$ ethanol solution for $30 \mathrm{sec}$. Seeds were dipped in sodium hypochlorite solution for 3 min. Between each wash the seeds were thoroughly washed with sterile distilled water and dried properly on autoclaved paper towels.

MS Media was prepared according to the standard protocol (Murashige and Skoog 1962). Different concentrations of chitosan (5, 10 and 15, $20 \mathrm{mg} /$ ) were dissolved in 1: 1 solution of $0.1 \mathrm{M}$ acetic acid and $0.2 \mathrm{M}$ sodium chloride solution. Solution was placed in shaker incubator overnight to dissolve the chitosan. $\mathrm{pH}$ of the solution was adjusted using $1 \mathrm{~N} \mathrm{NaOH}$ and added to the media. Medium without chitosan was used as control. Medium was autoclaved and allowed to cool. Sprouted Vigna radiata seeds were inoculated under aseptic conditions and grown for 15 - 20 days. All the trials were carried out in triplicates.

Growth of the seeds was observed regularly for 15 - 20 days and numbers of microbial colonies were counted. Averages of all the three trials were taken and arranged 
by completely randomized design (CRD). Data from in vitro cultures were subjected to ANOVA and means were compared using the critical difference (CD) test. Graph of number of microbial colonies $\mathrm{v} / \mathrm{s}$ concentration of chitosan was plotted. All the chemicals used for the experiment were purchased from Thermo fisher scientific Bangalore.

The growth of Vigna radiata seeds on the medium supplemented with different concentrations of chitosan is shown below. From Fig. 1 the control having no chitosan and lower concentrations of chitosan showed significant contaminations whereas $15 \mathrm{mg} A$ chitosan was found to have reduced contamination and optimum growth. Preliminary studies on the effect of chitosan were found to have growth enhancing effects as shown in Fig. 2. Similar results were obtained from the studies conducted by Sopalun et al. 2010 who worked on the effect of chitosan as a growth stimulant under in vitro conditions using Grammatophyllum speciosum, wherein half strength MS was supplemented with various concentrations of chitosan.



Fig. 1. Photographs with red circles are indication of contaminations. A. Control (0 $\mathrm{mg} /$ chitosan), B. $5 \mathrm{mg} /$ chitosan, C. $10 \mathrm{mg} /$ chitosan, D. $15 \mathrm{mg} /$ chitosan, E. $20 \mathrm{mg} /$ chitosan.

Fig. 3 represents the endophytic contamination in terms of the number of microbial colonies for different treatments in replicates, respectively. The endophytic contamination was high in case of control and cultures with lower concentrations when compared to the higher concentrations of chitosan. Considerable reduction in endophytic contamination was observed in $15 \mathrm{mg} /$ and it was found to be optimum.

Interpretation of the data using analysis software indicated that there was significant difference between the treatments. 15 and $20 \mathrm{mg} /$ of chitosan showed significant 


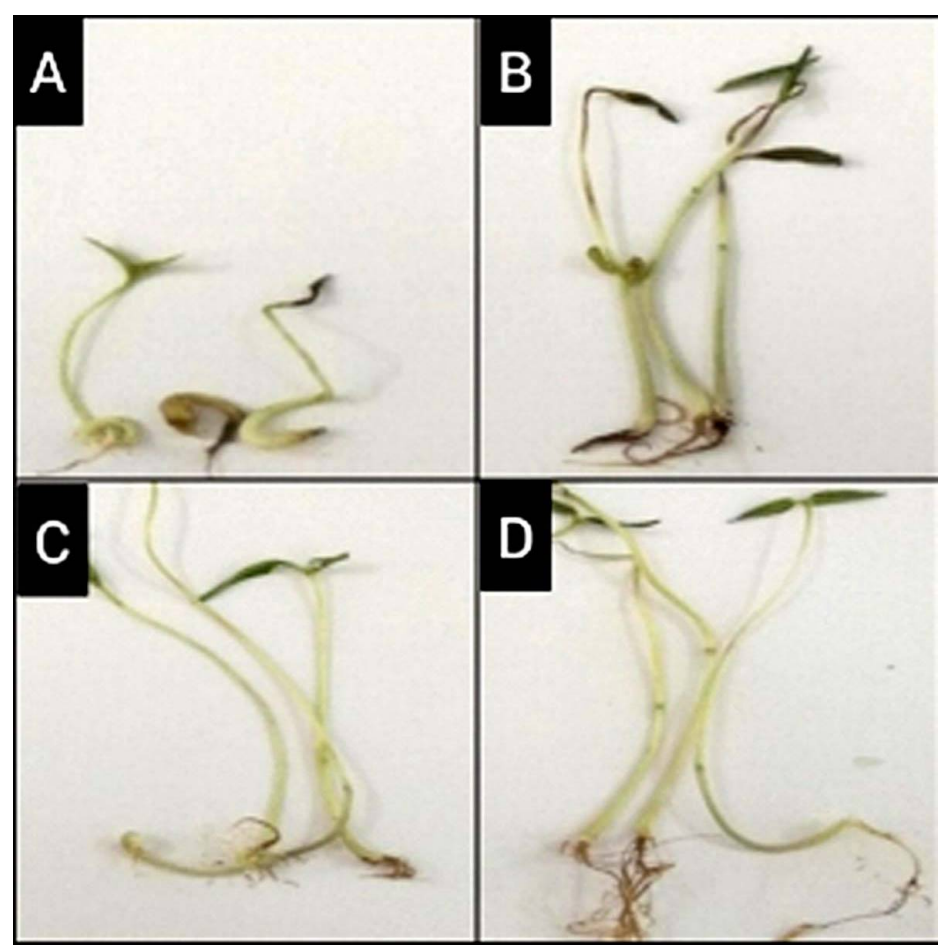

Fig. 2. Preliminary studies showing the effect of chitosan on the growth of Vigna radiata plantlets. A. $5 \mathrm{mg} /$ chitosan, B. $10 \mathrm{mg} /$ chitosan, C. $15 \mathrm{mg} /$ chitosan, D. $20 \mathrm{mg} /$ chitosan.

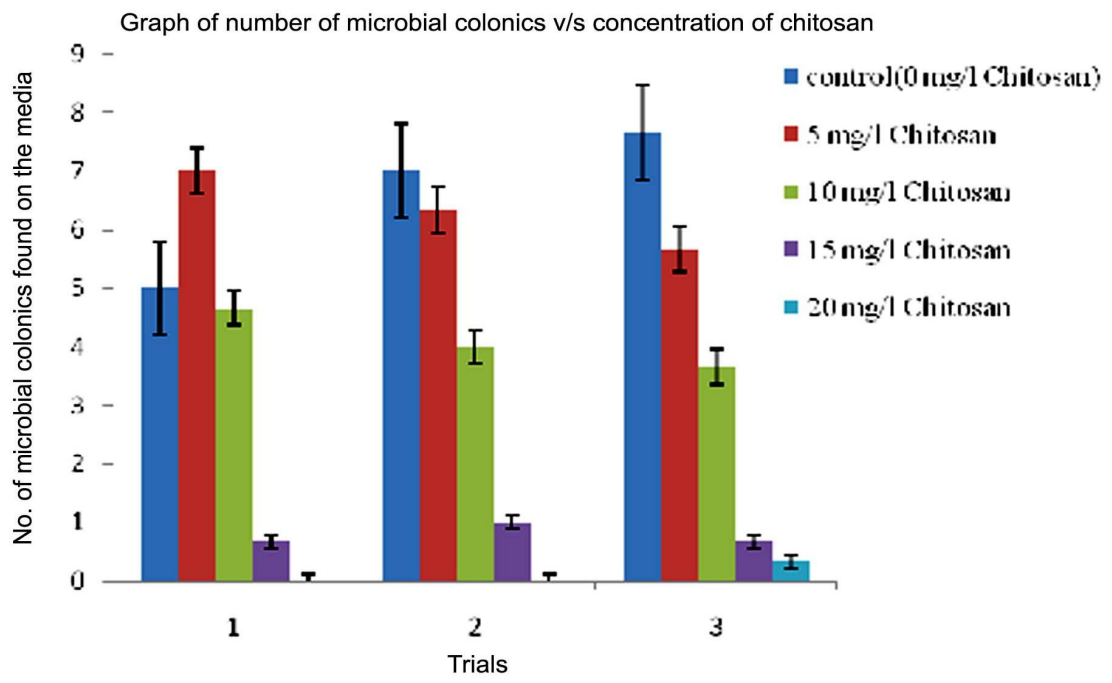

Fig. 3. Graph of number of microbial colonies $\mathrm{v} / \mathrm{s}$ concentration of chitosan showing reduced microbial growth on $15 \mathrm{mg} /$ concentration of chitosan 
results in comparison to the control and other treatments (Table 1). Endophytic contamination was found to be prominent in control. Fifteen $\mathrm{mg} /$ concentrations had lower level of contamination. Hence, chitosan can be used at $15 \mathrm{mg} /$ as a cost effective means to avoid endophytic contamination under in vitro conditions.

Table 1. ANOVA table for number of microbial colonies v/s concentration of chitosan

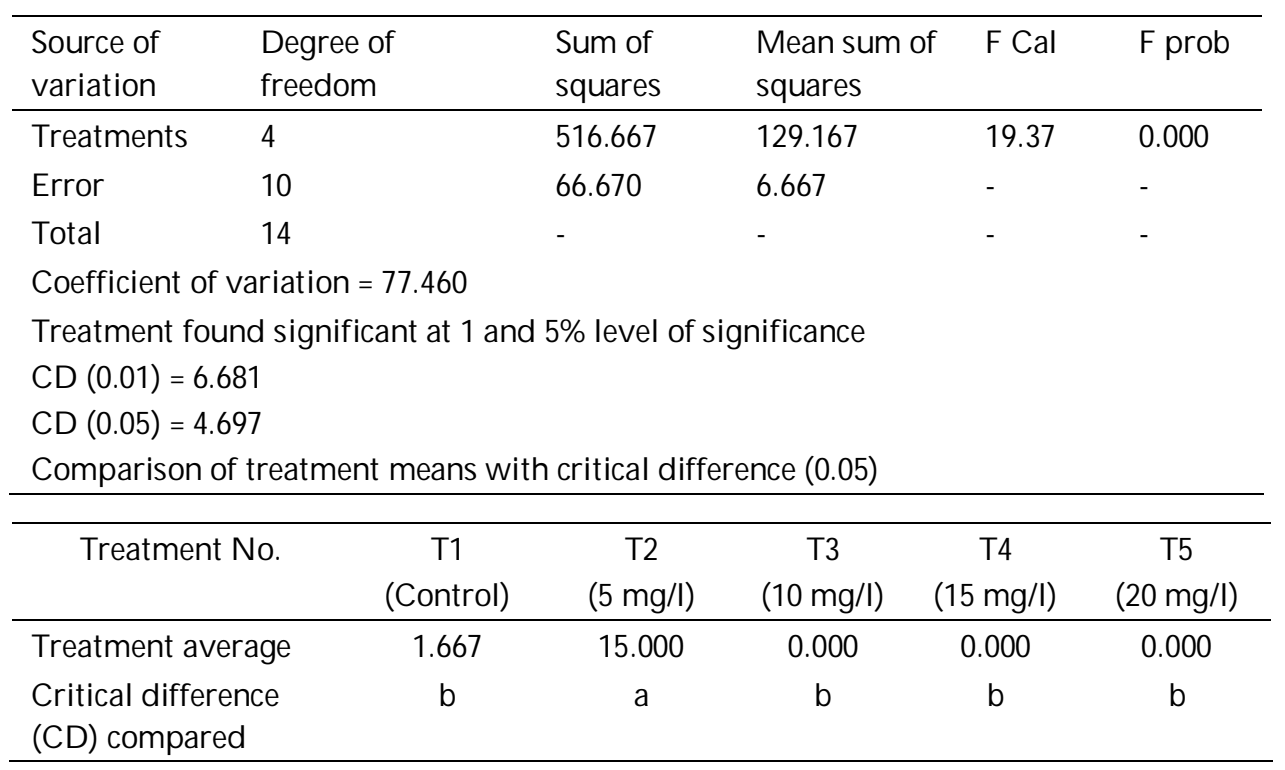

Table 2. Cost comparisons of various antibiotics and chitosan. (obtained from the catalogue of Thermo fisher scientific).

\begin{tabular}{lll}
\hline Sl. No. & Antibiotic & Price $($ Rs. $/ 10 \mathrm{mg})$ \\
\hline 1 & Ampicillin & 146.8 \\
2 & Gentamycin & 10162 \\
3 & Neomycin & 17.829 \\
4 & Kanamycin & 9.396 \\
5 & Penicillin & 3.468 \\
6 & Streptomycin & 621.4 \\
7 & Chitosan & 0.0195 \\
\hline
\end{tabular}

The cost of cheapest chitosan available is Rs. $1,950 / \mathrm{kg}$. The cost varies from 1,950 to Rs. $5,4485.7 \mathrm{~kg}$ depending upon the purity. So, chitosan can be used as a cost effective means to avoid endophytic contamination under in vitro conditions. 
Chitosan can be used as low cost substitute which is also known for its growth enhancing effects to control endophytic contaminations for in vitro culture of plants and avoid side effects of using antibiotics.

\section{Acknowledgement}

The authors are thankful to the Principal, Head of the Department of Biotechnology, R.V. College of Engineering Bangalore, for providing the lab facilities to carry out this study.

\section{References}

Ahmed El Ghaouth, Joseph Arul, Alain Asselin and Nicole (1992) Antifungal activity of chitosan on post-harvest pathogens: induction of morphological and cytological alterations in Rhizopus stolonifera, Mycological Research, 96(9): 769-779.

Cassells AC (1991) Problems in tissue culture: culture contamination. In: Debergh P.C.,Zimmerman R.H. (eds) Micro propagation. Springer, Dordrecht 31(1-14).

Falkiner F.R. (1997) Antibiotics in Plant Tissue Culture and Micro propagation - What are We Aiming at?. In: Cassells A.C. (eds) Pathogen and Microbial Contamination Management in Micropropagation. Developments in Plant Pathology, vol 12. Springer, Dordrecht, 155-160

Kneifel W and Leonhardt W (1992) Plant Cell Tissue Organ Culture, journal of plant biotechnology 29: 139.

Raafat D and Sahl HG (2009). Chitosan and its antimicrobial potential- A critical literature survey. Microbial Biotechnology 2(2): 186-201.

Sharp RG (2013) A Review of the Applications of Chitin and Its Derivatives in Agriculture to Modify Plant-Microbial Interactions and Improve Crop Yields. Agronomy 3: 757-793.

Sopalun K and Thammasiri KI (2010) Micropropagation of the Thai orchid Grammatophyllum speciosum Blume. Plant Cell, Tissue and Organ Culture 101(2): 143150. 